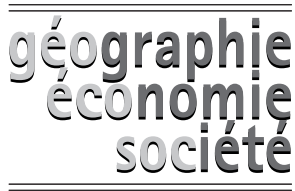

\title{
Quels indicateurs mobiliser pour les politiques environnementales locales ? Une approche à partir des services environnemen- taux et des conflits d'usage
}

\section{Which indicators can be used for local environmental policies? An approach with environmental services and land-use conflicts}

\author{
Olivier Aznara*, Marc Guérin ${ }^{\mathrm{b}}$, Philippe Jeanneaux ${ }^{\mathrm{c}}$ \\ Avec la collaboration de Céline Roche ${ }^{\mathrm{d}}$, Geneviève Bretiere ${ }^{\mathrm{e}}$, Serge Herviou ${ }^{\mathrm{f}}$ \\ ${ }^{a}$ ingénieur-chercheur - UMR Métafort Cemagref - 24 avenue des Landais - \\ BP 50085 - 63172 Aubière Cedex - tél .04 73440654 \\ ${ }^{b}$ chef de département - Cemagref, Département gestion des territoires - \\ Parc de Tourvoie - BP 44 - 92163 Antony Cedex - tel : 0140966034 - \\ ${ }^{c}$ Enseignant-chercheur - UMR Métafort ENITA - Département "Territoire \\ et Société"-Marmilhat - 63370 Lempdes - Tél : 04.73.98.70.30/13.24 \\ ${ }^{d}$ stagiaire Métafort Cemagref Clermont-Ferrand - ENSAIA Nancy \\ e assistant ingénieur - UMR Métafort Cemagref) \\ ${ }^{f}$ assistant ingénieur - Métafort Cemagref Clermont-Ferrand
}

\section{Résumé}

Face aux problèmes environnementaux locaux, les autorités publiques locales mettent en place des politiques environnementales ayant pour but l'entretien de l'environnement. Nous proposons d'utiliser le service environnemental comme un indicateur de réalisation des poli-

*Adresses email : Olivier.aznar@cemagref.fr•marc.guerin@cemagref.fr•jeanneaux@enitac.fr 
tiques environnementales locales. Nous suggérons en parallèle que le conflit d'usage est un indicateur d'alerte d'un problème environnemental. Nous analysons sur une période de 10 années sur la communauté de communes de Montrevel en Bresse (Ain) la production de services environnementaux et la survenance des conflits d'usage. Il ressort que les conflits d'usage mettent en évidence des problèmes environnementaux qui sont traités concrètement par de nombreux services environnementaux portés par différents acteurs.

(C) 2006 Lavoisier, Paris. Tous droits réservés.

\section{Summary}

In front of local environmental problems, local public authorities implement public policies to improve environment. We propose to use environmental service to reveal local environmental policies achievements. Further, we propose that land-use conflicts are relevant environmental problem signals. We analyse environmental service supplies and land-use conflicts during ten years on a French territory named Montrevel en Bresse (in departement Ain) that joins fourteen administrative districts. The empirical study provides useful insights about land-use conflicts related to environmental problems. We observe that environmental problems are solved by local authorities with many environmental services.

(C) 2006 Lavoisier, Paris. Tous droits réservés.

Mots clés : Service environnemental, conflit d'usage, indicateur, espaces ruraux et périurbains, politique publique locale.

Keywords: Environmental service, land-use conflict, indicator, rural and periurban areas, local public policies

\section{Introduction}

Les problèmes environnementaux concernant la détérioration de la qualité de l'eau, de l'air, des sols, les risques naturels et technologiques, les restrictions d'accès, ou encore la dégradation des milieux (Vernier, 2003; IFEN, 2002 ; PNUE, 2005), ont fréquemment une forte dimension locale. Afin de les traiter, les autorités publiques locales (communes, communautés de communes, syndicats intercommunaux), mettent en place des politiques environnementales fondées pour certaines sur l'entretien de l'environnement.

Pour préparer, suivre et évaluer ces politiques environnementales locales dans une logique de développement durable, le choix et la mise au point d'indicateurs pertinents constituent un facteur de réussite cruciale. Classiquement, l'étape de repérage de problèmes environnementaux est basée sur des indicateurs d'état qui rendent compte de la qualité de l'environnement. Il s'agit par exemple d'un taux de nitrate dans l'eau ou d'un niveau de biodiversité. Mais ces indicateurs ne semblent pas toujours suffisants. En effet, pour rendre compte des problèmes d'environnement, il est nécessaire d'intégrer la perception qu'en ont les acteurs locaux. Il existe souvent un décalage entre la mesure par des indicateurs techniques de l'évolution de l'environnement et la perception qu'en ont les acteurs. Lorsque la qualité de leur environnement ne les satisfait pas (ou plus) les usagers des ressources dégra- 
dés peuvent entrer en conflit. Sur la question du bruit des aéroports, par exemple, Faburel et Barraqué (2002) ont montré le hiatus existant entre les indicateurs de niveau sonore et la perception de la nuisance sonore par les acteurs locaux; tandis que Faburel (2003) a insisté sur la relation entre les indicateurs, le territoire et les conflits d'usage.

Aussi, pour compléter les indicateurs disponibles et en améliorer l'utilisation, nous posons la question suivante : quels indicateurs complémentaires est-il possible d'utiliser ? Il nous semble utile de mobiliser les conflits d'usage comme un indicateur d'alerte signalant les problèmes environnementaux qui se manifestent sur le territoire. En parallèle, nous proposons d'utiliser le service environnemental comme un indicateur de réalisation des politiques environnementales locales, car ils correspondent à des opérations concrètes d'entretien de l'environnement. L'apport central de l'article concerne les indicateurs de réalisation et d'alerte mobilisables pour la mise en œuvre ou l'ajustement des politiques environnementales locales.

Pour traiter de l'intérêt de ces indicateurs, notre propos s'organise autour des quatre étapes suivantes : dans une première partie, nous présentons le contexte de notre recherche centrée sur les politiques environnementales locales. Dans une deuxième partie, nous construisons notre cadre d'analyse des politiques environnementales locales. Dans la troisième partie, nous présentons la méthodologie d'une étude de cas des politiques environnementales conduites à l'échelle du canton rural de Montrevel en Bresse (Ain). La dernière partie présente les résultats de cette étude de cas.

\section{Contexte et problématique}

\subsection{Les problèmes environnementaux}

Ce premier point concerne les problèmes environnementaux et leur prise en compte exante par des indicateurs de qualité de l'environnement et de conflits d'usage.

Les indicateurs de qualité de l'environnement constituent des indicateurs d'état classiques sous le contrôle de l'expertise. Il s'agit souvent de comparaison entre un flux de pollution et une norme définie par les pouvoirs publics. Cette définition repose sur l'expertise scientifique. Le taux de nitrate dans l'eau représente un bon exemple : la norme a fixé à $50 \mathrm{mg} / \mathrm{l}$ d'eau le taux recommandé par les pouvoirs publics en France. Les mesures de qualité de l'eau sont conçues en regard de cette norme, fondée sur l'expertise et sur des arbitrages politiques.

Les conflits d'usage apparaissent comme des indicateurs originaux pour signaler un problème environnemental. Ils traduisent l'environnement tel qu'il est perçu par une partie (active et réactive) des acteurs locaux. Nous définissons, dans l'encadré $\mathrm{n}^{\circ} 1$, les conflits qui se développent dans les espaces ruraux. Ce développement est mis en exergue par Charlier, 1999 ; Perrier-Cornet, 2002 ; Torre, Caron, 2002 ; Lecourt, 2003 ; Jeanneaux, 2004 ; Torre et al, 2005. Ces travaux mettent en évidence les différents types de conflits qui se développent dans les espaces ruraux. 


\section{Encadré $\mathbf{n}^{\circ} 1$ : définition des conflits d'usage}

Nous définissons le conflit d'usage comme une situation d'opposition déclarée entre deux catégories d'agents dont les intérêts divergent à un moment précis lors de l'usage de biens localisés. Nous mettons l'accent sur les conflits ayant lieu dans l'espace rural et à dimension environnementale (Jeanneaux, 2004).

Nous étudions les conflits qui mettent en scène les pouvoirs publics (qu'ils soient contestés, contestataires ou arbitres lors d'un conflit) car ils nous semblent les plus intéressants à étudier pour faire le lien avec les problèmes environnementaux dans les espaces ruraux.

Notre grille d'analyse des conflits s'appuie sur la thématique sur laquelle portent les conflits et le bien-support associé. Nous avons regroupé les conflits en différentes thématiques environnementales telles que les conflits autour de la gestion de l'eau, des nuisances agricoles, de la gestion des déchets ou encore des problèmes au niveau des espaces récréatifs.

\subsection{Politiques environnementales locales : une action préventive ou correctrice}

Les politiques environnementales sont conçues pour prévenir ou corriger les situations estimées non durables sur le plan environnemental et correspondant à une préoccupation croissante d'une partie des citoyens. Elles font souvent écho au principe pollueur payeur, notamment à travers les taxes et les redevances. Mais les acteurs locaux développent également des actions d'entretien de l'environnement. A ce titre, ils développent des politiques environnementales locales reposant sur des théories d'action différentes. (cf. tableau 1).

Le tableau 1 présente une lecture des théories d'action sur lesquelles s'appuie l'intervention publique locale, dans le cadre de différentes politiques environnementales. Les actions peuvent être incitatives (payement direct au fournisseur, aide transitoire au pollueur, éducation, conseil et information) ou coercitive (principe pollueur payeur, mise sous tutelle publique).

\subsection{Un indicateur de l'action publique environnementale locale : le service environnemental}

Une modalité d'action des pouvoirs publics locaux en matière d'environnement souvent ignorée de la littérature économique concerne les actions d'entretien de l'environnement. Ces politiques environnementales locales sont de nature très diverse : elles peuvent concerner l'eau, l'air, les déchets, la biodiversité, les paysages, les pollutions ou encore les risques naturels. Elles donnent lieu, pour une partie importante d'entre elles, à des réalisations matérielles : il peut s'agir par exemple de la replantation de haies pour lutter contre l'érosion et améliorer les paysages, ou encore de l'installation d'une station d'épuration des eaux usées, ou bien d'une digue visant à lutter contre les inondations. Les exemples de réalisations sont très divers. 
Tableau 1 : théories d'action des politiques environnementales locales

\begin{tabular}{|c|c|c|c|c|}
\hline $\begin{array}{c}\text { Théories d'action } \\
\text { de la politique } \\
\text { environnementale }\end{array}$ & $\begin{array}{c}\text { Caractéristiques } \\
\text { du support } \\
\text { (bien ou individu) }\end{array}$ & $\begin{array}{c}\text { Réalisations } \\
\text { de la politique }\end{array}$ & $\begin{array}{c}\text { Exemple } \\
\text { de leviers } \\
\text { d'action }\end{array}$ & $\begin{array}{c}\text { Exemples } \\
\text { de politique }\end{array}$ \\
\hline $\begin{array}{l}\text { Gestion } \\
\text { des externalités } \\
\text { positives }\end{array}$ & Bien matériel & \multirow{3}{*}{$\begin{array}{l}\text { Production } \\
\text { de services } \\
\text { environnementaux }\end{array}$} & $\begin{array}{l}\text { Paiements } \\
\text { directs aux } \\
\text { fournisseurs }\end{array}$ & $\begin{array}{l}\text { Gestion } \\
\text { extensive } \\
\text { des prairies } \\
\text { et des haies }\end{array}$ \\
\hline $\begin{array}{l}\text { Gestion } \\
\text { des externalités } \\
\text { négatives }\end{array}$ & Bien matériel & & $\begin{array}{l}\text { Réponse } \\
\text { incitative : } \\
\text { aide transitoire } \\
\text { au pollueur, } \\
\text { par exemple } \\
\text { pour accélérer } \\
\text { la mise aux } \\
\text { normes des } \\
\text { installations } \\
\text { Réponse } \\
\text { coercitive : } \\
\text { principe pol- } \\
\text { lueur payeur }\end{array}$ & $\begin{array}{l}\text { Lutte contre } \\
\text { les pollutions } \\
\text { diffuses }\end{array}$ \\
\hline $\begin{array}{l}\text { Intervention directe } \\
\text { sur un bien } \\
\text { environnemental } \\
\text { localisé }\end{array}$ & Bien matériel & & $\begin{array}{l}\text { Gestion d'un } \\
\text { bien public } \\
\text { local }\end{array}$ & $\begin{array}{l}\text { Lutte contre les } \\
\text { risques naturels }\end{array}$ \\
\hline $\begin{array}{l}\text { Résolution } \\
\text { des problèmes de } \\
\text { droits de propriété }\end{array}$ & Bien matériel & $\begin{array}{l}\text { Planification spa- } \\
\text { tiale des usages }\end{array}$ & $\begin{array}{l}\text { Appropriation } \\
\text { privée ou en } \\
\text { club, mise } \\
\text { sous tutelle }\end{array}$ & $\begin{array}{l}\text { Plan local } \\
\text { d'urbanisme }\end{array}$ \\
\hline $\begin{array}{l}\text { Action } \\
\text { sur les représentations }\end{array}$ & $\begin{array}{l}\text { Individu } \\
\text { (usager final) }\end{array}$ & $\begin{array}{l}\text { Evolution } \\
\text { des préférences }\end{array}$ & $\begin{array}{l}\text { Education, } \\
\text { conseil, } \\
\text { information }\end{array}$ & $\begin{array}{l}\text { Sensibilisation } \\
\text { à l'environnement }\end{array}$ \\
\hline
\end{tabular}

Adapté de Guérin et al. (2001)

Comme toute politique publique, l'action des pouvoirs locaux dans le domaine de l'environnement doit être évaluée. L'évaluation des politiques environnementales prend une importance grandissante, comme en témoignent les travaux de Barraqué et Theys (1998), Lascoumes (1994) ou Barde (1991). Les travaux de sociologie, de science politique et d'économie se développent sur ce thème.

Nous proposons d'utiliser le concept de service environnemental comme un révélateur des actions concrètes d'entretien de l'environnement. Dans l'encadré $n^{\circ} 2$, nous définissons le service environnemental. 


\section{Encadré $n^{\circ} 2$ : définition des services environnementaux}

Nous retenons la définition suivante, en nous inspirant de l'économie des services (Gadrey, 2003) : un service environnemental désigne une contribution intentionnelle à la gestion d'un espace donné avec un but environnemental et collectif (Aznar, Perrier-Cornet, 2003 ; Aznar, Perrier-Cornet, 2004). Cette définition nécessite une traduction concrète. C'est pourquoi nous retenons quatre critères caractérisant un service environnemental :

- le premier concerne le bien-support sur lequel est produit le service environnemental. Il s'agit d'un espace naturel (autrement dit faiblement artificialisé). Il représente en quelque sorte un élément naturel constitutif du cadre de vie ;

- le deuxième critère représente l'acte technique, c'est-à-dire la modification du bien-support. Les actions correspondant à des valorisations (par exemple la réalisation de guides de randonnée), des études ou encore des zonages réglementaires ne répondent pas à la définition que nous proposons ;

- le troisième critère caractérise le service environnemental comme un acte volontaire, c'est-à-dire intentionnel.

- le dernier critère concerne la visée collective du service. Il s'agit donc d'un bien public au sens économique.

Ces 4 critères doivent être tous vérifiés pour qu'une action puisse être considérée comme un service environnemental.

Nous distinguons deux grandes catégories de services environnementaux : il s'agit d'une part de services environnementaux renouvelés qui sont produits tous les ans. D'autre part, il s'agit de services environnementaux ponctuels ou investissements environnementaux qui correspondent à des opérations réalisées à un moment donné.

\section{Cadre d'analyse}

Inspiré par le modèle d'analyse de politiques (Gibert, 1989; Parsons, 1995), notre cadre d'analyse présente quatre grandes séquences de l'intervention publique en matière d'environnement (Knoepfel et al., 2001) :

- la mise à l'agenda est la phase de formulation politique du problème public ;

- la programmation permet de déterminer les dispositifs et les acteurs choisis pour mettre en œuvre la politique ;

- la phase de mise en œuvre, se traduit concrètement par des réalisations financières et physiques s'adressant aux acteurs cibles. Dans notre recherche ces actions peuvent prendre la forme de services environnementaux

- la dernière étape correspond à l'évaluation et mesure les impacts de la politique.

Cet enchaînement séquentiel facilite le classement, l'analyse et présente un indéniable intérêt heuristique. Il serait évidemment illusoire de penser qu'il correspond à la réalité du processus de l'action publique. Comme l'énonçait déjà Gibert (1989), dans la réalité il n'est pas rare que l'ordre de la succession de ces différentes phases soit modifié et qu'il existe des problèmes sans dispositifs publics et à l'inverse des dispositifs d'intervention «à la recherche de problème». 
Pour apprécier l'exécution de chaque étape de la démarche, il est important de recourir à des indicateurs (Muller et Surel, 1998 ; Perret, 2001). C'est à ce niveau que nous proposons les services environnementaux (en tant qu'indicateurs de réalisation) et les conflits d'usage (en tant qu'indicateurs d'alerte d'un problème environnemental). Ces conflits peuvent naître de l'évolution de l'état de l'environnement, ils peuvent être également entretenus et amplifiés par la mise en œuvre de la politique publique.

La figure 1 présente notre cadre d'analyse de façon synthétique.

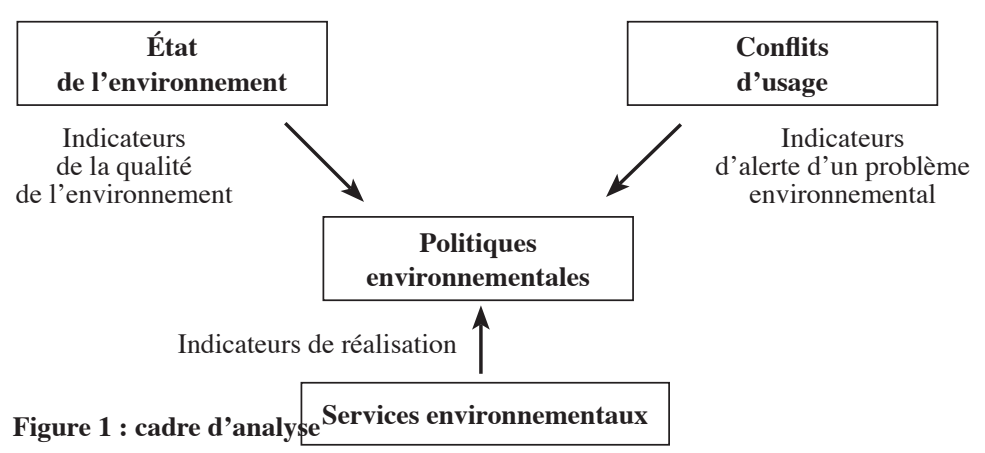

Il est important de ne pas confondre la méthode de construction d'un indicateur et sa méthode d'utilisation (qui consiste à lui donner du sens pour le décideur public ou l'évaluateur). Autrement dit, il faut distinguer l'information que donne l'indicateur de la définition de l'indicateur.

Après avoir précisé les méthodes de construction des indicateurs « conflit d'usage » (cf. encadré $\mathrm{n}^{\circ} 1$ ) et « service environnemental » (cf. encadré $\mathrm{n}^{\circ} 2$ ), nous pouvons maintenant proposer la méthode d'utilisation de nos indicateurs pour les politiques environnementales locales.

Le conflit d'usage, en tant qu'indicateur, a pour objectif d'apporte un éclairage sur la cause du problème à traiter et les acteurs incriminés et incriminants. Autrement dit, les conflits d'usage représentent des indicateurs d'un problème environnemental. Lorsque l'intensité conflictuelle augmente, l'alerte se déclenche et cela indique un problème environnemental à mettre à l'agenda politique. D'autres indicateurs de problèmes environnementaux sont mobilisés en parallèle. Il s'agit des indicateurs de qualité de l'environnement (normes, taux de pollution...), les problèmes étant identifiés ici à partir de diagnostics environnementaux. Dans la réalité, les deux types d'indicateurs (conflits et indicateurs de qualité) sont mobilisés de concert.

Le service environnemental, en tant qu'indicateur, a pour objectif d'apporter un éclairage sur les dimensions suivantes de l'action publique :

- l'objectif de la politique

- les fonds engagés (en euros)

- les acteurs locaux ou extérieurs concernés

Les services environnementaux ne recouvrent pas entièrement le spectre des réalisations issues de politiques environnementales. En particulier, les politiques foncières et les politiques réglementaires (dans le domaine de l'environnement) ne 
sont pas concernées par les services environnementaux. Mais notre indicateur de réalisation semble assez pertinent pour les politiques environnementales locales, qui prennent souvent la forme de subventions incitatives et donnent lieu à de nombreuses réalisations concrètes (ex : plantation de haies, restauration de berges de rivière, construction de station d'épuration).

\section{Méthodologie du recueil de données}

Le travail s'applique au canton de Montrevel en Bresse (Ain), un territoire rural périurbain qui présente une forte diversité d'usages. Dans ce canton, nous avons procédé à un inventaire aussi exhaustif que possible des services environnementaux et des conflits d'usage environnementaux (Roche, 2004). Nous avons également recueilli différents diagnostics portant sur la qualité de l'environnement (qualité de l'eau, risques d'inondation, localisation des haies, localisation des chemins de randonnée). Nous présentons les traits principaux du canton de Montrevel avant d'exposer notre méthode de recueil des données.

\subsection{Zone d'étude et échelle d'analyse}

Nous avons retenu une échelle cantonale pour simplifier le recueil des données, relativement lourd. Le canton de Montrevel, constitué en une communauté de communes regroupant 14 communes (cf. figure 2), constitue un bon archétype d'un espace périurbain encore fortement marqué par l'agriculture et où l'intercommunalité occupe une place structurante dans l'évolution de l'espace'. Ce territoire traditionnellement assez peuplé est traversé par la rivière Reyssouze. Celle-ci joue un rôle important sur ce territoire hydromorphe où l'espace «naturel » résulte d'une construction particulièrement minutieuse. La proximité d'autoroutes place le canton à seulement une heure de Lyon ou de Genève et à une quinzaine de kilomètres du pôle urbain de Bourg-en-Bresse. Ces dernières années, de nouveaux résidents, à la recherche d'un cadre de vie de qualité, sont arrivés sur le canton qui a connu une augmentation de population de $10 \%$ en 10 ans et compte aujourd'hui 12600 habitants. On observe une rapide modernisation de l'agriculture avec pour corollaire la disparition d'une partie du bocage bressan. Le canton est aussi doté d'une importante base de tourisme et de loisir (100 000 nuitées par an) située sur un plan d'eau. Ainsi, il s'agit d'un territoire aux usages diversifiés, bien adapté à la recherche d'un ensemble de services environnementaux variés.

\subsection{Méthode de recueil des données}

Nous avons retenu la décennie 1993-2002 car de nombreuses politiques environnementales ont démarré à partir de 1993 à la suite de décisions adoptées à l'échelle communautaire, française ou mondiale en 1992 (la loi française sur l'eau, la réforme de la PAC ou encore les résolutions de la conférence de Rio).

1 De manière générale, les facilités d'accès aux documents administratifs ont également conditionné notre choix. L'étude aurait pu porter sur un territoire plus étendu, la méthode étant reproductible, mais le temps disponible ne l'a pas permis. 


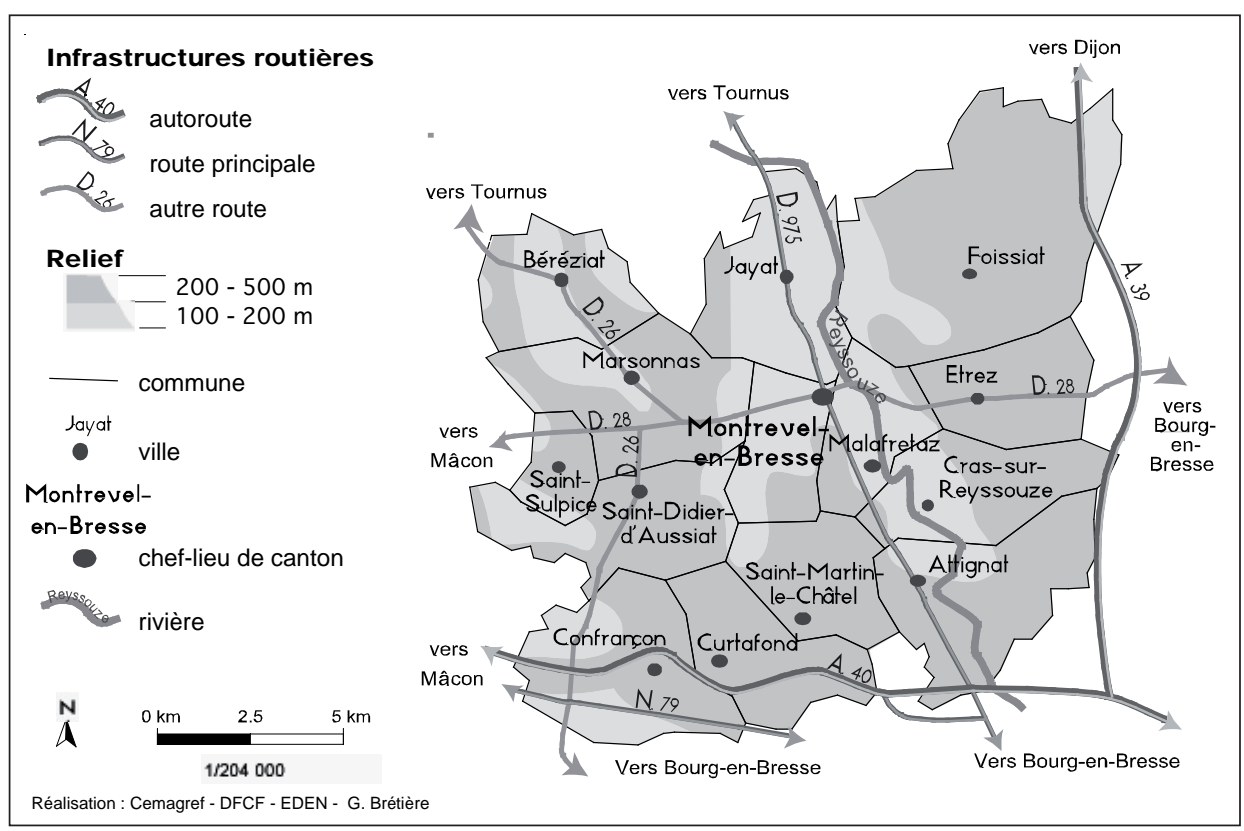

Figure 2 : Localisation de la zone bressane

La rivière Reyssouze est totalement régulée par une série de biefs, le paysage paraît soigneusement jardiné, l'attention apportée aux haies a longtemps occupé une place élevée dans les valeurs sociales locales. Aussi semble-t-il intéressant de s'interroger sur la traduction effective de ces politiques à l'échelle d'un territoire rural sur le moyen terme. Les services environnementaux sont recensés à partir des sources administratives (dossiers des politiques environnementales et budgets des communes) et des entretiens auprès des personnes-ressources des différents secteurs institutionnels. Un recoupement systématique des informations a été entrepris (au sein des sources écrites et entre sources écrites et orales).

Nous avons au total effectué une cinquantaine d'entretiens auprès de deux groupes d'acteurs. Nous avons tout d'abord interrogé les élus des 14 communes du canton et des établissements publics de coopération intercommunale (EPCI), ainsi que les techniciens des EPCI locaux (communauté de communes, syndicat de rivière). Nous avons effectué une vingtaine d'entretiens auprès des responsables d'associations porteurs d'usages différents (randonnée, vélo tout terrain, pêche, chasse, protection de la nature et valorisation du patrimoine). Il n'était pas possible de rencontrer l'ensemble des agriculteurs sur le canton, c'est pourquoi nous avons identifié leur contribution à travers l'examen de documents administratifs².

\footnotetext{
2 De plus, si un agriculteur effectue un service environnemental en dehors d'une politique, nous rencontrons le commanditaire potentiel de ce service (association ou élu), ce qui limite les risques d'oubli.
} 
Nous avons recensé les services environnementaux issus de divers dispositifs ${ }^{3}$ à partir de la consultation des documents auprès des organismes instructeurs et de ceux qui ont participé à la constitution des dossiers (préfecture de l'Ain, direction départementale de l'agriculture et de la forêt (DDAF) et conseil général). La logique contractuelle de ces politiques conduit à réaliser des dossiers détaillés qui fourmillent d'informations.

Les entretiens permettant difficilement d'aborder avec précision des services environnementaux, la consultation des budgets s'est avérée nécessaire (Aznar et al., 2002). En effet, l'importance économique de chaque service environnemental réalisé est souvent peu connue ou oubliée des élus. Néanmoins, l'exploitation des budgets est parfois difficile et demande beaucoup de précautions car ils présentent les dépenses de façon très agrégée. .

\subsection{Identification des conflits d'usage}

Les conflits sont recensés grâce aux entretiens auprès des élus et personnes-ressources de chaque commune. Ils ont été également recueillis à partir de l'examen de la presse quotidienne régionale (éditions locales du Progrès de l'Ain sur la période 20012002). Les sources écrites et orales sur les conflits ont été systématiquement recoupées. Cependant, nous n'avons pas retenu les conflits d'usage concernant la concurrence foncière car cela représentait une démarche trop lourde et ces conflits nous semblaient avoir un lien relativement indirect avec les politiques environnementales.

\section{Résultats de l'étude empirique'}

Cette partie a pour objet de présenter les résultats principaux de l'étude empirique, en essayant de les mettre en perspective par rapport à la réflexion sur les indicateurs.

\subsection{L'identification des problèmes environnementaux à partir d'indicateurs}

Nous avons identifié quatre grands problèmes environnementaux. Il s'agit respectivement de la pollution de la Reyssouze, des inondations, de la disparition du bocage bressan et du développement des loisirs verts.

\subsubsection{Problèmes de pollution de la rivière}

Indicateurs de qualité de l'environnement

La Reyssouze est un cours d'eau fortement dégradé (indice de pollution «moyen à fort $»$ concernant les nitrates et le phosphore ${ }^{4}$.

\footnotetext{
${ }^{3}$ Contrat territorial d'exploitation (CTE), prime au maintien des systèmes d'élevage extensif (PMSEE), fonds de gestion de l'espace rural (FGER), programme de maîtrise des pollutions d'origine agricole (PMPOA), Leader II, programme de développement rural (PDR) dans le cadre de l'objectif 5b puis de l'objectif 2, programme local de gestion de l'espace (PLGE) financé par le conseil régional Rhône-Alpes.

4 Source : District de Montrevel-en-Bresse, ACEIF, 2001, Diagnostic territorial et orientations stratégiques, Phases 1 et 2 de l'agenda 21 local (sensibilisation, diagnostic et orientations), Montrevel-en-Bresse, 129 p.
} 


\section{Indicateur d'alerte}

Des pollutions telles que les rejets par une usine d'équarrissage en amont du canton, le fonctionnement défectueux de la station d'épuration d'Attignat ont été les causes majeures de la mauvaise qualité des eaux de la Reyssouze. Huit conflits se sont déclenchés suite à des pollutions accidentelles « visibles ».

\subsubsection{Problèmes d'inondations}

La rivière Reyssouze, profondément aménagée, a en plusieurs endroits un lit majeur plus haut que les prairies avoisinantes.

Indicateur de qualité de l'environnement

Les inondations sont fréquentes dans la zone d'étude. Nous n'avons pas pu disposer d'un indicateur quantitatif concernant les inondations.

Indicateur d'alerte

Les inondations de lotissements provoquent des conflits d'usage entre les résidents et les communes (presque une dizaine de conflits).

\subsubsection{Problèmes de disparition du bocage}

Ce problème est apparu à l'agenda politique local dans les dix dernières années dans le canton de Montrevel en Bresse.

\section{Indicateur de qualité de l'environnement}

La disparition du bocage bressan constitue un problème qui préoccupe plus les élus que les habitants interrogés. Le bocage est aujourd'hui réduit à une faible densité, désorganisé et vieilli. Nous n'avons pas pu obtenir d'indicateur chiffré de la densité du bocage. C'est pourquoi nous mobilisons une proxie : la surface en maïs qui augmente régulièrement pour atteindre 3600 ha en 2000 (source : recensement de l'agriculture 2000).

Indicateur d'alerte

Les conflits à propos du bocage concernent à la fois sa destruction volontaire et les conditions de production du service environnemental de replantation. Ce dernier est encouragé par des incitations financières (PLGE, CTE) mais certains agriculteurs sont opposés à la replantation.

\subsubsection{Problèmes autour du développement des loisirs verts}

Le dernier grand problème environnemental du canton de Montrevel touche aux espaces de loisirs verts.

Indicateurs de qualité de l'environnement

Ce problème ne fait pas référence à une disparition, une pollution ou un risque naturel comme les précédents. Il n'existe pas d'indicateur de qualité de l'environnement.

Indicateurs d'alerte

Huit conflits d'usage concernent la gestion des chemins de randonnée et des espaces de loisirs verts, indiquant les problèmes autour des loisirs verts.

\subsection{Indicateurs de réalisation de la politique environnementale locale : les services environnementaux}

Lors de la phase d'enquête, 551 services et investissements environnementaux ont été recensés sur le canton de Montrevel au cours de la période 1993-2002. Ce nombre donne 
simplement une idée de l'importance de ces services environnementaux dans un canton rural. Nous essayons d'articuler ces services environnementaux avec les théories d'action qui les sous-tendent.

\subsubsection{Lutte contre la pollution}

Pour juguler la pollution de l'eau, les acteurs locaux ont initié des services environnementaux en grand nombre qui concernent d'une part l'assainissement et l'épuration des eaux, compétence classique des communes et de l'intercommunalité et, d'autre part, la lutte contre la pollution agricole (concentrée ou diffuse). Les investissements environnementaux (tels que les stations d'épuration) s'élèvent à 17 millions d'euros sur 10 ans. L'assainissement (16 millions d'euros) représente la majeure partie de ces dépenses portées par les communes et le syndicat de rivière avec des subventions importantes du conseil général, de l'agence de l'eau et de l'Etat. Les services environnementaux renouvelés (dépenses de fonctionnement) représentent 700000 euros par an.

La théorie d'action qui sous-tend cet investissement environnemental relève de la gestion d'un bien public local avec des subventions publiques externes au territoire local. L'étude de l'indicateur de qualité de l'environnement (issu des documents de suivi de la qualité de l'eau de la rivière) montre que la pollution a baissé. Parallèlement, le nombre de conflits autour de la gestion des eaux usées a diminué au cours de la période d'étude.

Concernant les pollutions de l'eau d'origine agricole, 1400000 euros ont été investis dans la construction de fosses à lisier et 162000 euros par an dans des services environnementaux d'adaptation des pratiques agricoles (implantation de cultures intermédiaires, travail du sol simplifié, lutte biologique, bandes enherbées). L'intervention publique en ce domaine consiste à subventionner temporairement le pollueur pour respecter les règles. L'efficacité de ces services environnementaux est relativement médiocre en raison d'une inadéquation entre l'échelle du problème (bassin versant) et celle du service environnemental conçu au niveau d'une exploitation agricole. L'absence de coordination à l'échelle du territoire concourt à expliquer cette faible efficacité.

Notons l'émergence, depuis 1998, d'investissements environnementaux dans la restauration de la ripisylve par le syndicat de rivière, pour un montant global de 100000 euros. Il s'agit là d'un nouveau type de bien public environnemental, que gèrent les collectivités locales depuis le milieu des années 1990. Les compétences ne sont pas clairement définies sur les berges de rivière (propriété privée des berges, mais action publique de plus en plus développée de la part des collectivités locales et d'autres acteurs publics) car les droits d'usage et les droits de propriété ne se recoupent pas parfaitement.

La collectivité se substitue aux propriétaires défaillants (dans ce canton comme dans de nombreuses zones en France). Apparaît ici le potentiel conflictuel des services environnementaux : la restauration de la ripisylve, si elle est conduite pour lutter contre la pollution (et atténuer les conflits autour de la pollution de l'eau), conduit à des conflits avec certains propriétaires riverains qui s'opposent à cette prise en charge publique. Tout se passe comme si les pouvoirs publics abordaient de plus en plus la rivière comme un bien sous tutelle, sans pour autant maîtriser le droit de propriété sur ce bien. Ces conflits sont cependant tempérés par le développement de procédures de concertation concernant la gestion des cours d'eau, tels les contrats de rivière (Brun, Marette, 2003) ou les schémas d'aménagement et de gestion des eaux (SAGE). 


\subsubsection{Lutte contre les inondations}

Des investissements de l'ordre d'un million d'euros sur 10 ans ont été réalisés sur le canton pour répondre à ce problème. Seul le syndicat de rivière est intervenu par des actions de curage de biefs et d'automatisation de vannes-clapet. Ces actions s'amplifient à partir de 1998, avec la mise en place du contrat de rivière. On peut observer une réduction du nombre d'inondations mais une dizaine de conflits d'usage (qui prennent la forme de plaintes de riverains contre les communes) existent encore. Du point de vue économique, la lutte contre les risques s'apparente au principe économique de la gestion d'un bien public local, mais sans la maîtrise du droit de propriété sur ce bien.

\subsubsection{Maintien du bocage}

Le volume financier consacré aux services environnementaux de maintien du bocage est plus modeste que pour les deux précédentes politiques environnementales. Il atteint environ 57000 euros. Ces investissements se répartissent équitablement entre les agriculteurs dans le cadre des CTE et la communauté de communes dans le cadre du programme local de gestion de l'espace (PLGE). Au total, $13 \mathrm{~km}$ de haies ont été plantés.

La question de l'entretien du bocage est cruciale, au-delà de la replantation de haies. A ce niveau, les communes dépensent 98000 euros par an pour l'entretien des haies. Les dépenses des agriculteurs pour l'entretien des haies n'ont pas pu être calculées, faute de données. Mais nous disposons de données pour l'entretien plus global des prairies : les agriculteurs qui assurent la gestion extensive des prairies reçoivent des aides publiques d'un montant annuel de 130000 euros sur le canton de Montrevel, attribués dans le cadre des mesures agro-environnementales. Ces aides sont dispersées en un grand nombre d'opérations d'un faible montant. L'intérêt de la préservation des prairies est généralement souligné par les experts environnementaux (par exemple Cozic et al. 1999). Mais cette gestion extensive des prairies n'est pas zonée sur le canton étudié en fonction d'enjeux prioritaires.

L'implication des collectivités locales (communes et communauté de communes) traduit une volonté de gestion d'un bien public local. Ces dernières agissent sur des espaces emblématiques (par exemple le long de sentiers communaux) sans pouvoir toujours privilégier l'efficacité environnementale.

\subsubsection{Amélioration de la qualité des espaces de loisirs verts et touristiques,}

Les flux financiers des services environnementaux mis en œuvre pour répondre à ce problème sont assez faibles (466 000 euros d'investissements environnementaux et 350000 euros pour les services environnementaux renouvelés), par rapport aux problèmes autour des rivières. Deux grandes catégories d'acteurs, la communauté de communes et les communes interviennent, par la création et l'entretien de sentiers de randonnée et des espaces verts. Il faut souligner la place des associations de randonneurs, qui réalisent un travail non négligeable de gestion des sentiers de randonnée. Le principe économique est celui de la gestion d'un bien commun aux usages multiples. Les services environnementaux peuvent permettre de répondre aux problèmes autour des chemins de randonnée, mais ils suscitent aussi de nouveaux conflits, en particulier de la part de riverains qui s'opposent au passage de chemins de randonnée sur leurs terrains. 


\subsection{Synthèse de l'étude empirique}

Il apparaît de nombreux services environnementaux, en nombre croissant sur les 10 années étudiées, pour les 4 catégories de problème. Les trois-quarts des dépenses ont pour origine les collectivités locales, avec l'aide de nombreuses subventions. La moitié des services environnementaux sont réalisés par les agriculteurs, mais ils ne représentent toutefois que le quart du montant financier total.

\section{Conclusion}

En réponse à la question posée en introduction, les services environnementaux représentent de bons indicateurs de réalisation de politiques environnementales locales. Les conflits d'usage se révèlent de bons indicateurs d'alerte signalant les problèmes environnementaux, en complément des indicateurs de qualité de l'environnement. Il nous semble important de construire un appareil statistique adapté pour produire ces nouveaux indicateurs, afin d'améliorer la préparation, le suivi et l'évaluation des politiques environnementales locales.

Le même type d'étude pourrait être réalisé sur d'autres cantons afin d'établir des comparaisons, ou à une plus grande échelle (étude de départements par exemple) dans l'objectif d'une généralisation des données. Pour cela, il faudrait cibler un seul type de service, tel que la gestion des haies ou la gestion du milieu aquatique. Mais il convient de creuser le questionnement dans d'autres directions. Une question importante concerne l'adéquation entre les conflits d'usage et les problèmes environnementaux, mais son traitement nécessite un couplage avec des évaluations environnementales, afin de comparer les résultats apportés par les indicateurs de qualité de l'environnement et ceux apportés par la recension des conflits d'usage.

\section{Références}

Aznar, O., Perrier-Cornet, P., 2003. Les services environnementaux dans les espaces ruraux : une approche par l'économie des services. Économie rurale 273-274, janvier-avril, 142-157.

Aznar, O., Perrier-Cornet, P., 2004. The production of environmental services in rural areas: Institutional sectors and proximities. International Journal of Sustainable Development 7 (3) p. 257-272.

Barde, J.-P., 1991. Économie et politique de l'environnement, Presses universitaires de France, Paris, 383 p.

Barraqué, B., Theys, J. (dir.), 1998. Les politiques d'environnement : évaluation de la première génération, 1971-1995. Éd. Recherches, Paris, 391 p.

Brun, A., Marette, S., 2003. Le bilan d'un contrat de rivière : le cas de la Reyssouze. Économie rurale 275, $30-50$.

Charlier, B., 1999. La défense de l'environnement : entre espace et territoire, géographie des conflits environnementaux déclenchés en France depuis 1974, thèse de doctorat. Université de Pau et des pays de l'Adour, département de géographie, $753 \mathrm{p}$. 
Cozic, P, Thiébaud, F., Bernard-Brunet, J., Bornard, A., Brau-Nogué, C., Dobremez, L., Ernoult, C., Favier, G., Paisant, J.-P., Véron, F., 1999, Évaluation des effets environnementaux de la prime au maintien des systèmes d'élevage extensifs, Rapport final de synthèse, Cemagref, département gestion des territoires, unité de recherche agricultures et milieux montagnards, Grenoble, juin, 214 p.

Faburel G., Barraqué B. (2002), Les impacts territoriaux du bruit des avions. Le cas de l'urbanisation à proximité de Roissy CDG. Ne pas évaluer pour pouvoir tout dire, et son contraire, Rapport final pour l'ADEME, dans le cadre du programme Concertation, Décision et Environnement du MATE, 43 p.

Faburel G., 2003, «Le bruit des avions, facteur de révélation et de construction des territoires », l'Espace Géographique, n³, pp. 205-223.

Gadrey, J., 2003. Socio-économie des services. Repères, la Découverte, Paris, 128 p.

Guérin, M., Aznar, O., Candau, J., Le Floch, S., Michelin, Y., Moquay, P., 2001. Acteurs locaux et initiatives publiques dans le domaine du paysage - Une analyse du processus de construction des interventions publiques localisées, Rapport intermédiaire du projet $n^{\circ} 16$ Tranche 99-1, Programme de recherche MATE « Politiques Publiques et paysages «. Cemagref - ENITAC - ENGREF, 32 p.

IFEN, 2002. L'environnement en France. La Découverte, Paris.

Jeanneaux, P., 2004. Economie de la décision publique et conflits d'usages dans les espaces ruraux et périurbains. Communication aux 4èmes journées de la proximité. Marseille, 17 et 18 juin, 26 p.

Lascoumes, P., 1994. L'éco-pouvoir : environnements et politiques. La Découverte, Paris, 320 p.

Lecourt A., 2003, Les conflits d'aménagement: analyse théorique et pratique à partir du cas breton, thèse de doctorat en géographie, aménagement de l'espace-urbanisme, université de Rennes 2 Haute-Bretagne, 361 p.

Muller, P., Surel, Y., 1998. L'analyse des politiques publiques, collection «clefs politiques». Montchrestien, Paris, $156 \mathrm{p}$.

PNUE, , collectif, 2005, Rapport de synthèse de l'évaluation des écosystèmes pour le millénaire, ONU, 59 p.

Perret, Bernard, 2001, L'évaluation des politiques publiques, la Découverte, Paris, 127 p.

Perrier-Cornet, P. (dir.), 2002. À qui appartient l'espace rural ? Enjeux publics et politiques. Editions de l'Aube/ DATAR, La Tour d'Aigues, $141 \mathrm{p}$.

Roche, C., 2004. Analyse des services environnementaux et mise en relation avec les enjeux des espaces ruraux - Etude de cas sur le canton de Montrevel-en-Bresse (Ain), mémoire de fin d'études. Cemagref ClermontFerrand, ENSAIA Nancy, 35 p.

Torre A., (dir.), Aznar O., (dir.), Aviles A., Bonin M., Caron A., Guérin M., Jeanneaux P., Kirat T., Lefranc C., Melot R., Thinon P., Bretière G., Herviou S., 2005, Une analyse des modalités de résolution des tensions liées aux différents usages des espaces ruraux, Rapport final, Programme de recherche pour et sur le développement régional - INRA Rhône-Alpes, 130 p.

Torre, A., Caron, A., 2002. Conflits d'usages et de voisinage dans les espaces ruraux. Sciences de la société 57, octobre, 95-113.

Vernier, Jacques, 2003, L'environnement, Presses Universitaires de France, Paris, 127 p. 
